\title{
IA Aplicada a Jogos Digitais: Oportunidades e Desafios no Campo da Educação
}

\author{
Eduardo Aranha1, Wendell Araújo ${ }^{1}$, Charles Madeira ${ }^{2}$ \\ ${ }^{1}$ Departamento de Informática e Matemática Aplicada - Universidade Federal do Rio \\ Grande do Norte (UFRN) - Natal - RN - Brasil \\ ${ }^{2}$ Instituto Metrópole Digital - Universidade Federal do Rio Grande do Norte (UFRN) - \\ Natal - RN - Brasil \\ eduardoaranha@dimap.ufrn.br, wendell.cmdegmail.com, charles@imd.ufrn.br
}

\begin{abstract}
Educational Games have an important role in education. Nevertheless, there are several challenges that need to be faced by long term research projects. In this way, the artificial intelligence (AI) presents itself as a research area with great potential of support to overcome these challenges. In this article, we present a review of the literature on digital games in education and how AI has been used to support digital games in general. Based on this, we present the main challenges for digital educational games and how AI can be used in long-term research to solve them.
\end{abstract}

Resumo. Jogos digitais educativos têm um papel importante na educação. Entretanto, existem vários desafios que precisam ser enfrentados com pesquisas de longo prazo para se alcançar seu pleno uso e benefícios esperados. Neste sentido, a inteligência artificial (IA) apresenta-se como uma área de pesquisa com grande potencial de apoio para superar esses desafios. Neste artigo, apresentamos uma revisão da literatura sobre jogos digitais na educação e como a IA já vem sendo utilizada na área de jogos digitais, de forma geral. Com base nisso, apresentamos os principais desafios para os jogos digitais educacionais e como a IA pode ser utilizada em pesquisas de longo prazo para solucioná-los.

\section{Introdução}

A inteligência artificial (IA) vem avançando em passos largos com o objetivo de tornar as tarefas dos seres humanos mais produtivas e eficazes. Esses avanços têm proporcionado o desenvolvimento de diversas aplicações e equipamentos que são cada vez mais presentes no cotidiano. Apenas para exemplificar, temos sistemas inteligentes nos smartphones, nos imóveis, nos automóveis, nos robôs, soluções para monitoramento de tarefas de risco, entre outros. Isto pode ser percebido em diversos setores tais como a saúde, a educação, o comércio, a indústria, o exército, etc. No contexto da educação, particularmente, diversas iniciativas vêm sendo desenvolvidas com técnicas de IA. Um exemplo bastante impressionante neste sentido é o sistema Watson da IBM $^{1}$, que é

\footnotetext{
${ }^{1}$ https://www.ibm.com/watson/education
} 
empregado em muitas aplicações educacionais nos diversos níveis de ensino a fim de facilitar o processo de ensino-aprendizagem [King et al. 2016].

Por outro lado, com o intuito de tentar solucionar o grave problema da falta de motivação e da participação dos estudantes da atualidade, diversos pesquisadores têm fomentado a utilização de jogos na sala de aula. A área conhecida como Game-based Learning [Prensky 2007] surge como uma metodologia de ensino baseada em técnicas lúdicas de aprendizagem juntamente com ações de brincadeira inclinados à realidade dos alunos. Aprender jogando é a mola mestra desta metodologia que tem demonstrado ser eficiente no ensino das mais diversas disciplinas presentes nos currículos das escolas e universidades uma vez que os jogos se mostram como a linguagem nativa dos jovens da geração atual. Os jogos são, desse modo, um importante instrumento pedagógico, facilitador da aprendizagem e da consolidação de conhecimento, e que oferecem aos alunos uma forma prazerosa, cativante, motivadora e divertida de trabalhar conteúdos. Mais que uma simples animação do fazer pedagógico ou simplesmente uma forma de aprendizagem mais interativa, os jogos desenvolvem diversas habilidades e competências nos alunos.

Quando unimos esses dois mundos, da IA com os jogos, apresentamos uma grande área de pesquisa que vem aumentando consideravelmente o seu nível de importância na comunidade científica e na indústria. Por um lado, o interesse fundamental dos pesquisadores em IA pelos jogos vem do fato que eles se mostram como um ótimo laboratório de experimentação para pôr em prática e avaliar as técnicas desenvolvidas. Por outro lado, as técnicas de IA permitem aumentar o grau de realismo dos jogos, assim como o grau de imersão dos jogadores, tornando a experiência do ato de jogar cada vez mais agradável. Isso pode ser constatado através de inúmeras pesquisas conforme publicações efetuadas em grandes conferências da área tais como a IEEE Conference on Computational Intelligence and Games e a AAAI Conference on Artificial Intelligence and Interactive Digital Entertainment.

No entanto, apesar do forte potencial da IA para tornar os jogos cada vez mais envolventes para os jogadores, esta área de pesquisa ainda não é uma direção que vem sendo explorada com interesse pela comunidade científica no contexto dos jogos para educação. Portanto, este artigo visa apresentar diversos direcionamentos de pesquisa em IA no contexto dos jogos para educação a fim de incentivar os pesquisadores a se engajarem na busca de soluções para os grandes desafios existentes.

\section{Revisão da literatura}

Esta seção apresenta inicialmente uma revisão da literatura acerca do uso dos jogos digitais na educação e, em seguida, mostra como a inteligência artificial vem sendo aplicada nos jogos digitais voltados ao entretenimento para se resolver diferentes tipos de problemas.

\subsection{Jogos digitais educacionais}

Os jogos digitais possuem um grande potencial para a área educativa, [Savi e Ulbricht 2008] apontam que eles apresentam um efeito motivador, facilitador de aprendizado, socialização, aprendizado por descoberta ao tentar resolver problemas durante o jogo e 
que um aluno pode se tornar experiente em um determinado conteúdo ao se tornar experiente em um jogo que aborde esse mesmo conteúdo.

[Victal et al. 2015] ressalta a importância de que os jogos devem ser parte integrante das disciplinas e não como um acessório da modernidade e que o professor deve estar preparado para aplicar um novo formato de aulas com a inclusão dos jogos. $\mathrm{O}$ autor também aponta que é possível incluir o desenvolvimento de jogos na disciplina, porém é necessário que faça a utilização de uma game engine simples de fácil uso para não frustrar os alunos pela dificuldade da ferramenta. Os participantes do estudo aplicado também ressaltaram que os jogos são ferramentas importantes de aprendizagem, bem como a sua utilização na educação e que todo o processo ajuda no desenvolvimento da capacidade cognitiva de quem o realiza.

[Dias et al. 2017] realizam uma busca do uso de jogos digitais na educação básica e constataram que os jogos trazem uma contribuição além do conteúdo abordado, como o letramento digital, raciocínio lógico, aumento no engajamento, compreensão conceitual. De fato, os jogos atuam como uma ferramenta que aproxima os alunos dos professores. Outro ponto que pode ser notado pelo trabalho é a carência de publicações na área além da falta de uso de referências pedagógicas para o desenvolvimento.

Poucas são as aplicações de IA que estão sendo utilizadas e abordadas voltadas para a educação. Conforme observado em [Araújo et al. 2016) poucas publicações foram encontradas do uso de adaptação de jogos no contexto educativo. Além disso, não é encontrado qual a melhor forma de usar e em que tipo de conteúdo estão sendo focados, pois ainda é uma ferramenta pouco explorada pelos demais. Ainda assim, com os trabalhos aplicados um dos principais benefícios apontados foi a motivação gerada para deixar os alunos mais interessados. Além disso, verificou-se que o aprendizado dos alunos foi mais eficiente utilizando os métodos adaptativos criados pela IA.

\subsection{Inteligência artificial aplicada a jogos digitais}

A IA é uma das principais partes durante o desenvolvimento de um jogo, podendo ser utilizada de diversas formas como na movimentação de um objeto, controle de fala de personagens, criação automática de mapas entre outros. Assim, ela se tornou um recurso para ser aplicado e testado em busca de diversas soluções para os jogos.

Os custos que envolvem o desenvolvimento de um jogo são altos, gerando assim a busca por soluções que tentem reduzir o custo para desenvolver o mesmo. [Togelius et al. 2011] fala sobre a geração procedural de conteúdo (PCG) que é a criação de conteúdo realizada através de algoritmos e com isso ajudar a reduzir a produção de conteúdo para um jogo, pois a máquina seria responsável por criar o necessário para o jogo. Com isso, alguns outros benefícios são encontrados conforme [Korn et al. 2017]: a agilidade na produção; redução dos custos; alto valor de replay; proporcionar novas experiências ao usuários.

Um outro desafio que é encontrado nos jogos é a questão do balanceamento do nível de dificuldade, pois há uma variedade muito grande de jogadores sendo alguns mais experientes que outros e sempre é difícil manter um jogo desafiador para determinados usuários. [Wheat et al. 2015] mostra como pode ser realizada um ajuste de dificuldade dinamicamente (DDA) realizando um estudo com um jogo de plataforma 
procurando deixar o jogo sempre equilibrado independentemente do nível de habilidade do jogador fazendo uso de agentes. [Lopes e Bidarra 2011] apresentam um survey mostrando as diversas aplicações para adaptabilidade em jogos não só para adaptação de nível, mas para adaptação de frustração, ansiedade ou tédio buscando sempre um estado de diversão ideal para cada jogador. Tal adaptação pode ser realizada através da extração do desempenho do jogador durante a execução do jogo. Assim, a extração desses dados pode servir para diversas aplicações dependendo do que será aplicado, podendo descobrir se o jogador está evoluindo à medida que está jogando, se ela não está conseguindo avançar ou entender determinado problema encontrado no jogo.

Além disso, a IA auxilia no desenvolvimento de diversas outras soluções. Por exemplo, [Liapis et al. 2013] apresentam o Sentient Sketchbook, uma ferramenta para auxiliar o designer durante a produção de um cenário de jogo. Ela cria esboço de mapas em baixa resolução, testa o mapa para restrições de jogabilidade além de avaliar as propriedades do mapa a ser criado, promovendo assim a colaboração entre o designer e o software. Já outros trabalhos tratam da geração automática de sons musicais para jogos, AI players capazes de testar e avaliar cenários de jogos, entre outras funcionalidades.

\section{Desafios na área de jogos digitais educacionais e oportunidades de aplicação da inteligência artificial}

Esta seção apresenta os principais desafios encontrados no desenvolvimento de jogos digitais educacionais. Cada um dos desafios é descrito individualmente e é acompanhado por uma discussão sobre que ações em termos de pesquisa em inteligência computacional podem ser realizadas no contexto dos jogos para se trabalhar cada desafio ao longo da próxima década.

\subsection{Recursos reduzidos para o desenvolvimento dos jogos}

Embora a indústria de jogos digitais movimente mais recursos que a indústria de cinema, este volume de recursos é aplicado principalmente em jogos de entretenimento. Isto porque o setor educacional não é tão atrativo em termos comerciais, por ser um ambiente de difícil incorporação de novas tecnologias e de investimentos reduzidos por parte dos governos municipais, estaduais e federal, e das próprias escolas privadas. Equipes de projetos de jogos educacionais geralmente são pequenas, muitas vezes financiadas por projetos de pesquisa com recursos limitados.

Neste sentido, pode ser investigado como fazer uso das técnicas de geração procedural de conteúdo para gerar não só o cenário e demais elementos de entretenimento do jogo, mas também os elementos relacionados aos objetivos de aprendizado desejados. Algoritmos evolutivos, baseados em gramática e deep learning são formas de se implementar esses mecanismos de geração automática de conteúdo.

\subsection{Avaliação formativa}

O professor de uma escola geralmente trabalha com turmas grandes, com dezenas alunos, e precisam estar realizando avaliações formativas para poder realizar intervenções mais eficazes. Essas avaliações feitas de forma tradicional tomam muito tempo (corrigir provas, etc.). Os jogos digitais educacionais nesse contexto podem 
contribuir para realizar avaliações de forma mais massiva, gerando dados sobre os estudantes a cada partida.

Entretanto, para se realizar avaliações formativas alguns desafios precisam ser melhor trabalhados. Entre eles, temos questões de como modelar as habilidades e competências desejadas para se trabalhar no jogo e como relacioná-las a ações dos jogadores dentro do jogo. Além disso, existem habilidades curriculares (matemática, português, etc.) e habilidades esperadas para os profissionais do século XXI que precisam ser trabalhadas e avaliadas (resolução de problemas, colaboração, persistência, etc.).

Redes bayesianas vem sendo utilizadas para se modelar as habilidades desejadas para o estudante e seu nível de proficiência atual em cada uma delas. Entretanto, existem desafios relacionados a como se modelar automaticamente desafios no jogo que trabalhem adequadamente essas habilidades, ou de se identificar automaticamente a partir de um jogo já existente que registros de game log fornecem evidências sobre uma habilidade específica. Técnicas de mineração de dados e de sequência de eventos podem ser investigadas para realizar análises automáticas nas grandes massas de dados gerados pelos jogos digitais. Esses registros podem gerar evidências tanto sobre conhecimentos curriculares como sobre habilidades cognitivas.

\subsection{Engajamento e adequação do nível de dificuldade do jogo}

Na sala de aula tradicional, embora cada estudante tenha suas limitações e necessidades específicas, preferências e ritmo de aprendizado próprio, o professor trabalha o ritmo de suas aulas conforme o desempenho médio da turma. Nos jogos digitais, isso pode ser trabalhado de forma otimizada, desde que existam modelagens do conhecimento e perfil do estudante.

Nos jogos de entretenimento, a adaptabilidade e o uso de recompensas nos jogos são bastante trabalhados de forma a maximizar o interesse do jogador no jogo e o tempo que o mesmo passa jogando-o. Para os jogos digitais, pode-se investigar mecanismos de adaptabilidade que façam uso de modelos do aprendizado do estudante para implementar sistemas de recomendação ou de parametrização da geração procedural do conteúdo dos jogos. Isso irá tornar os jogos mais balanceados em termos de dificuldades relacionadas às questões mais pedagógicas, além do balanceamento tradicional do nível de dificuldade de mecânicas de entretenimento do jogo.

O engajamento dos estudantes no jogo pode ser melhorado através do desenvolvimento de personagens interativos inteligentes capazes de detectarem as emoções dos jogadores e ajustarem o nível de dificuldade do jogo ou interagirem de forma a reconhecer as conquistas do jogador e encorajá-los a enfrentar os desafios mais difíceis. Sensores do meio físico e virtual podem ser utilizados para gerar dados a serem analisados por técnicas de aprendizado de máquina e criarem modelos de predição de emoções.

\subsection{Autoria de jogos por professores e alunos}

O sistema de ensino brasileiro possui um número muito grande de áreas de conhecimentos, componentes curriculares e habilidades a serem aprendidas. É um 
desafio para a área de jogos digitais conseguir cobrir as partes principais dessas habilidades a serem aprendidas. Para atenuar esse problema, existem diversas iniciativas para apropriar o professor e seus alunos com ferramentas de construção de jogos digitais com fins educativos. Esta abordagem permite não só o professor e seus alunos criarem jogos para temas ainda não trabalhados, mas também permite um processo mais ativo, criativo e participativo dos estudantes. Para isso, porém, existem ainda desafios como a falta de preparo técnico dos professores e alunos para a atividade de desenvolvimento de jogos digitais.

Nesse contexto, pode ser investigado o uso de técnicas de geração procedural de conteúdo guiada por parâmetros definidos pelos professores e alunos, visando o desenvolvimento de jogos que trabalhem temas definidos como de interesse. Além disso, pode-se ser desenvolver agentes inteligentes que funcionem como assistentes virtuais de game design, co-criando o game design através de recomendações realizadas a partir da proposta sendo desenvolvida pelo estudante. Estas recomendações podem ser baseadas no perfil do autor humano e na base histórica de game designs desenvolvidos, por exemplo.

Outra linha de pesquisa a ser trabalhada é a extração de informações de textos (narrativas, etc.) escritos pelos estudantes em documentos de game design para geração semiautomática dos jogos. Técnicas de processamento de linguagem natural precisam ser adaptadas para o contexto de game design de jogos em escolas do ensino básico.

Para desenvolver jogos atrativos, os mesmos devem possuir imagens visualmente interessantes de personagens, inimigos, cenários, etc. Técnicas como deep learning podem ser usadas para criar variações de imagens em bases de dados existentes e informações do perfil dos estudantes. Por exemplo, pode-se investigar mecanismos que mudem as cores dos personagens e cenários de acordo com um dado tema ou cenário de fundo. Ou que novos inimigos ou heróis sejam criados a partir da combinação de componentes já existentes.

A geração de sons para os jogos também é um desafio que pode ser trabalhado pela aplicação de técnicas que combinem sons de instrumentos básicos, ajustando-se o volume e intensidade dos sons de acordo com o nível de dificuldade atualmente enfrentado pelo jogador. Cenários requerendo respostas mais rápidas podem exigir músicas mais eletrizantes, enquanto cenários que desafiam a mente podem requerer sons mais suaves.

\subsection{Feedback sobre jogos criados por professores e alunos}

Uma vez que é permitido aos professores e alunos a criação de jogos digitais como atividade acadêmica, é importante para um melhor processo de aprendizagem se ter mecanismos para informar aos autores sobre a qualidade dos jogos produzidos.

Algoritmos de busca de soluções podem ser utilizados para se desenvolver mecanismos que informem a viabilidade do jogo, como por exemplo ter ou não solução. Mecanismos de análise de retrospectiva (replay) podem ser aplicados para que os criadores dos jogos saibam como seus desafios estão sendo solucionados ou não pelos estudantes. 
Agentes inteligentes que assumem o controle do herói do jogo no lugar do jogador também podem ser desenvolvidos para simular partidas considerando diferentes perfis de habilidades. Por exemplo, ter agentes que simulem o comportamento provável no jogo de estudantes com baixa ou com alta proficiência em uma determinada habilidade de interesse. Essas simulações podem ser aprendidas por exemplo a partir da análise do log de partidas de jogadores com diferentes níveis de conhecimento. $\mathrm{O}$ desafio se torna ainda maior ao se tentar desenvolver agentes que consigam jogar diferentes tipos de jogos, ou até aprender sozinho a jogar um jogo do qual não foi treinado (AI player genérico).

\subsection{Ampliar aprendizado através da mentoria}

A interação entre estudantes é bastante importante para o aprendizado dos mesmos. Dentre as interações possíveis, temos a de estudante, ele opina e tira dúvidas de outros estudantes, como em um processo de mentoria. Este processo permite um maior aprofundamento do entendimento da questão pelo estudante e pode ser utilizado dentro dos jogos digitais educativos.

Agentes inteligentes podem ser modelados para que aprendam com o estudante. Este aprendizado pode ser feito de diferentes formas. Uma primeira forma é observar e aprender automaticamente o comportamento do estudante, reproduzindo seu conhecimento nas partidas. Assim, o estudante poderá visualizar seu próprio comportamento e refletir sobre ele.

Outra forma que pode ser investigada é a do agente ir perguntando ao estudante como resolver desafios e ir aprendendo com suas respostas. Os estudantes trabalhariam como mentores (ou treinadores) dos agentes, que poderiam ser colocados para disputar desafios em competições ou simplesmente servirem para estimular o estudante a raciocinar e expressar seu conhecimento para o agente.

Esses agentes podem também ser trabalhados para realizar sugestões para os estudantes, incentivando o pensamento crítico do estudante sobre suas ações e fazendo-o refletir sobre como melhorar seu conhecimento e suas habilidades.

\subsection{Balanceamento do aprendizado cooperativo e competitivo}

Dependendo da situação, pode ser interessante para o professor disponibilizar aos seus alunos ambientes de aprendizado colaborativo e ambientes que promovam a competição entre eles. Nos jogos multiplayers, jogadores podem cooperar para atingir um objetivo em comum ou competir para ver quem consegue ir mais longe no jogo, ou ganhar mais pontos, por exemplo. Este processo pode ser delicado e possui desafios como o de encontrar estudantes com perfis apropriados para trabalharem juntos, seja de forma colaborativa ou de forma competitiva, de acordo com seus níveis de conhecimentos escolares relevantes para a atividade educacional apresentada pelo jogo.

Técnicas de inteligência artificial podem ser investigadas e aplicadas para a modelagem dos jogadores e de oponentes, buscando adversários reais (seres humanos) ou virtuais (agentes inteligentes) com habilidades semelhantes ou complementares, promovendo assim um trabalho colaborativo ou uma disputa mais justa, de acordo com o interesse do professor. 


\subsection{Explorar mundo físico}

A aprendizagem significativa ocorre quando novas informações são apresentadas baseadas em conhecimentos prévios do estudante. Uma das formas de se ocorrer esse tipo de aprendizagem dentro dos jogos é incluir dentro dos jogos não só conhecimentos prévios, mas também o contexto da realidade vivenciada pelo aluno ou do mundo de forma geral em que vivemos.

Técnicas de inteligência artificial podem ser aplicadas nesse contexto para fazer uso da realidade virtual ou realidade aumentada, inserindo assim elementos do mundo real no jogo. Reconstruções de terrenos desmatados, por exemplo, poderiam ser utilizados para se trabalhar temas de recuperação ambiental ou processo de desmatamento.

\subsection{Infraestrutura laboratorial reduzida}

A realidade das escolas brasileiras é de maneira geral retratada pela falta de infraestrutura adequada de laboratórios de informática, computadores atualizados e outros tipos de equipamentos. Por outro lado, os próprios professores e estudantes possuem a sua disposição celulares smartphones e tablets pessoais, que poderiam ser utilizados quando necessário. Nesse sentido, é um desafio para a área de jogos digitais educativos conseguir desenvolver soluções que possam ser aplicadas nesse contexto, com poucos equipamentos eletrônicos (tablets e smartphones) e com mais materiais físicos disponíveis (quadro branco, papéis, etc.).

Técnicas de inteligência artificial e de visão computacional podem ser utilizadas para se investigar como desenhos de personagens, cenários e outras informações poderiam ser capturadas por dispositivos móveis e inseridas dentro dos jogos. Dessa forma, o professor teria condições de trabalhar em larga escala com material usualmente encontrado nas escolas e alguns poucos tablets de origem da escola ou dos próprios estudantes.

\section{Considerações finais}

Este artigo apresenta o importante papel dos jogos digitais educacionais para a educação e vários desafios para o desenvolvimento e aplicação dos mesmos no setor educacional, principalmente no contexto do Brasil. Uma revisão da literatura destaca que a comunidade de jogos digitais educacionais é ativa, e que muito ainda pode ser feito quando se comparado às pesquisas relacionadas a jogos digitais de maneira geral (entretenimento, principalmente).

São apresentados neste trabalho um conjunto com nove desafios para os pesquisadores e para a indústria de jogos digitais educacionais, os quais são discutidos caminhos que podem ser trilhados a partir da aplicação de pesquisas da área de inteligência artificial, aliadas a pesquisas da área de educação e de desenvolvimento de jogos digitais de forma geral. Algumas destas pesquisas já possuem alguns resultados e encaminhamentos nos jogos de entretenimento puro, precisando ainda serem investigadas e adaptadas às suas aplicações para o setor educacional. Outros desafios 
requerem pesquisas ainda em estágio embrionário, mesmo quando considerado as outras áreas de aplicação dos jogos digitais.

Através dos desafios e oportunidades apresentadas por este trabalho, espera-se estar contribuindo para que grupos de pesquisas na área de jogos digitais educacionais possam definir novos rumos e trabalharem na fronteira do estado da arte, trazendo contribuições significativas para a educação ao longo da próxima década.

\section{Referências}

Araújo, W. O., Silva, T. R., Aranha, E. H. S. (2016) “Aplicação de Jogos Adaptativos na Educação: Uma Revisão Sistemática da Literatura”, In: XXVII Simpósio Brasileiro de Informática na Educação.

Dias, J. L., Talita, L., Medeiros, H. B., Aranha, E. H. S. (2017) "O Uso de Jogos Digitais na Educação Básica: uma Revisão Sistemática da Literatura”, In: XVI Simpósio Brasileiro de Jogos e Entretenimento Digital.

King, M., Cave, R., Foden, M., Stent, M. (2016) "Personalised education: From curriculum to career with cognitive systems". IBM Education.

Korn, O., Blatz, M., Rees, A., Schaal, J., Schwind, V., Gorlich, D. (2017) "Procedural Content Generation for Game Props? A Study on the Effects on User Experience", In: Computers in Entertainment (CIE) - Theoretical and Practical Computer Applications in Entertainment, Nr. 15(2).

Liapis, A., Yannakakis, G. N., Togelius, J. (2013) "Sentient Sketchbook: Computer-Assisted Game Level Authoring", In: Proceedings of the 8th Conference on the Foundations of Digital Games (FDG).

Lopes, R., Bidarra, R. (2011) "Adaptivity Challenges in Games and Simulations: a Survey", In: IEEE Transactions on Computational Intelligence and AI In Games, 3(2), 85-99.

Prensky, M. (2007) “Digital Game-Based Learning”. McGraw-Hill.

Savi, R., Ulbricht, V. R. (2008) "Jogos Digitais Educacionais: Benefícios e Desafios”, In: Revista Novas Tecnologias na Educação - RENOTE, v.6, n.2.

Victal, E. R. N., Junior, H. A. P., Rios, P. T. G, Menezes, C. S. (2015) "Aprendendo Sobre o Uso de Jogos Digitais na Educação”, In: XXI Workshop de Informática na Escola.

Togelius, J., Kastbjerg, E., Schedl, D., Yannakakis, G. N. (2011) "What is Procedural Content Generation?: Mario on the Borderline", In: Proceedings of the 2nd Workshop on Procedural Content Generation in Games.

Wheat, D., Masek, M., Lam, C. P., Hingston, P. (2015) "Dynamic Difficulty Adjustment in 2d Platformers through Agent-based Procedural Level Generation" In: IEEE International Conference on Systems, Man, and Cybernetics. pp. 2778-2785. 